\title{
ROLE OF THE MICROTUBULE-ASSOCIATED TPPP/p25 IN PARKINSON'S AND RELATED DISEASES AND ITS THERAPEUTIC POTENTIAL
}

\begin{abstract}
Introduction: The discovery and development of therapeutic strategies for the treatments of Parkinson's disease (PD) and other synucleinopathies are limited by a lack of understanding of the pathomechanisms and their connection with different diseases such as cancers.

Areas covered: The hallmarks of these diseases are frequently multifunctional disordered proteins displaying moonlighting and/or chameleon features, which are challenging drug targets. A representative of these proteins is the disordered Tubulin Polymerization Promoting Protein (TPPP/p25) expressed specifically in oligodendrocytes (OLGs) in normal brain. Its non-physiological level is tightly related to the etiology of PD and Multiple System Atrophy (TPPP/p25 enrichment in inclusions of neurons and OLGs, respectively), multiple sclerosis (TPPP/p25-positive OLG destruction), as well as glioma (loss of TPPP/p25 expression). The established anti-proliferative potency of TPPP/p25 may raise its influence in cancer development. The recognition that whereas too much TPPP/p25 could kill neurons in PD, but its loss keeps cells alive in cancer could contribute to our understanding of the interrelationship of "TPPP/p25 diseases".

Expert commentary: The knowledge accumulated so far underlines the key roles of the multifunctional TPPP/p25 in both physiological and diverse pathological processes, consequently its validation as drug target sorely needs a new innovative strategy that is briefly reviewed here.
\end{abstract}

\section{KEYWORDS}

$\alpha$-synuclein, cancer, CNS diseases, drug target, microtubule, protein chameleon, tubulin deacetylases, Tubulin Polymerization Promoting Protein/p25, validation.

\section{INTRODUCTION: PRESENT STATE OF PARKINSON RESEARCH}

Parkinson's disease (PD), the second most common neurodegenerative disorder belongs to the family of synucleinopathies, which includes dementia with Lewy bodies (LBD) and multiple system atrophy (MSA). Classic motor signs of PD can be attributed mainly to the substantial loss of dopaminecontaining neurons in the substantia nigra pars compacta [1]. L-Dopa, dopamine agonists and amantadine as well as new MAO-B and catechol-o-methyltransferase inhibitors appear to be efficacious treatments of motor complications [2,3]. Indeed, L-Dopa appears as the most effective therapy, it is considered as a "gold-standard"; however, after 4-6 years the patients frequently suffer from severe side effects such as dyskinesia [4]. During the past decades pharmacotherapy, deep brain stimulation and physiotherapy have been evaluated for the symptomatic treatment of PD as well. The major problem with the pharmacotherapy is the lack of drugs that halt the progression of the disease. There are new drugs and other therapies such as glia cell line-derived neurotrophic factor (GDNF); however, these treatments failed in phase 3 trials to translate the successful results from preclinical to clinical studies $[3,4]$ due to the application of inappropriate preclinical models (1-methyl-4-phenyl-1,2,3,6tetrahydropyridine (MPTP), 6-hydroxydopamine) as well as targets often representing late stage of PD [4].

In the recent years, the Parkinson research on new drugs focuses on $\alpha$-synuclein (hallmark protein of the disease), its small oligomers and related proteins [4]. $\alpha$-synuclein is involved in the

URL: https://mc.manuscriptcentral.com/eru Email: anna.woolman@informa.com 
control of neurotransmitter release and re-cycling as well as in synaptic and structural plasticity [5]; its aggregation can affect synaptic function and axonal firmness, which is supported by the observation that neuronal susceptibility in PD is related to axonal length, axonal caliber and the degree of myelination [5]. Immunizations against $\alpha$-synuclein and inhibitors or modulators of $\alpha$-synuclein aggregation are under clinical trials [4]. The disordered $\alpha$-synuclein is degraded mainly by the ubiquitin-proteasome system, whereas more complex conformations, including aggregates, are removed by the autophagylysosomal pathway [6-8]. In fact, pathogenic depletion of proteasome components and lysosomes observed in brains of PD patients has underlined the notion that defects in the protein quality-control mechanism contribute to PD pathogenesis [9-10].

Progressive neuronal cell loss and widespread aggregation of $\alpha$-synuclein forming Lewy bodies and Lewy neurites are the major characteristics of PD and other synucleionopathies [11]. After the initial appearance of Lewy neurites, additional aggregates are formed and rapidly accumulate leading to oligomers, protofibrils and finally insoluble Lewy bodies and Lewy neurites [12]. Although the staging/categorization systems (such as Braak's staging) are appropriate in most cases; a significant number of patients with widespread $\alpha$-synuclein aggregation classified in Braak stage 5-6 do not have a diagnosis or symptoms of PD [13,14]. In fact, until the recent past the $\alpha$-synuclein aggregate was considered as a major drug target; nowadays it seems to be justified that the small, soluble oligomeric forms are regarded as the fatal species [15], the assembly/co-assembly of which leads to the formation of aggregates. In this complex process the mutations of $\alpha$-synuclein also play a role. The identification of mutations in the gene encoding $\alpha$-synuclein (SNCA) in families with PD had a substantial effect on PD research [16]. Clinical mutations of human $\alpha$-synuclein are Ala53Thr, Ala30Pro, Glu46Lys, His50Gln, Gly51Asp and Ala53Glu; and the presence of two major phosphorylation sites, Ser87 and Ser129 in Lewy bodies has been reported to be characteristic for PD pathology [17,18]. However, it is well documented that wild type $\alpha$-synuclein aggregates were detected in the brain inclusions of patients suffering from sporadic PD, which accounts for about $90 \%$ of all cases [3]. In order to prevent/arrest the pathological assembly of $\alpha$-synuclein, the not-yet discovered mechanism driving the etiology of PD needs to be established, in this relation the microtubule system is a key player.

The microtubule network plays crucial roles in diverse processes such as cell division, differentiation, intracellular trafficking as well as in pathological inclusion body formation; its dynamic instability is central to these functions allowing them to rapidly reorganize, differentiate spatially and temporally in accordance with environmental signals/factors $[19,20]$. The microtubules, their major posttranslational modifications (tyrosination, acetylation) and microtubule associated proteins (parkin, PTEN induced putative kinase 1 (PINK1), leucine-rich repeat kinase 2 (LRRK2) and $\alpha$-synuclein) regulate long-distance intracellular cargo trafficking along axons and dendrites; the dysfunction of the interplay between cytoskeletal components is reflected in PD. The fragmentation of stable microtubules and the depletion of dynamic ones coupled with impairment of axonal transport seem to be common in the early phases of the pathology of synucleinopathies [21,22]. In PD experimental models, an earlier microtubule destabilization followed by the enrichment of acetylated and detyrosinated microtubules and the block of axonal transport were often observed. Microtubules are necessary in pathological inclusion body formation [23], since aggregated protein species are delivered to the aggresome by a retrograde transport on microtubules [24]. However, the relationship between microtubules and $\alpha$ synuclein is complicated $[21,22]$. Tubulin can promote $\alpha$-synuclein fibrillation in vitro, but there are conflicting data on whether destabilization of the microtubule network facilitates or prevents the aggregation in vivo, or $\alpha$-synuclein influences microtubule formation. Recently $\alpha$-synuclein has been found as a foldable microtubule dynamase regulating nucleation and dynamics of microtubules [25]. 
In conclusion, to date the exact mechanisms of progressive dopaminergic cell loss remain to be unraveled. It is a real question what induces aggregation of $\alpha$-synuclein. Lewy bodies contain a variety of molecular components such as tubulin, microtubule associated proteins, ubiquitin, heat shock and autophagosomal proteins [26] and Tubulin Polymerization Promoting Protein (TPPP/p25) [27-30] that could be the initiator of $\alpha$-synuclein aggregation. Another important, but not well-studied aspect of PD pathology is the concomitant beta-amyloid and/or tau aggregates in a significant number of patients $[14,31,32]$. No disease-modifying treatment is available [21,33,34], consequently, therapeutic development should focus on the hallmark proteins. In reality, there is a real stagnation in the development of new drugs. The crucial bottleneck is the lack of primary endpoints, which reflect the progression of PD when the substantia nigra is not or only very mildly affected. Our new innovative strategy to validate an anti-Parkinson drug target makes attempt to contribute to this issue.

\section{MOONLIGHTING AND CHAMELEON FEATURE OF TPPP/p25}

TPPP/p25 has been identified besides $\alpha$-synuclein as hallmark of PD and synucleinopathies [2729]. It does not have a well-defined $3 \mathrm{D}$ structure; it is an intrinsically disordered protein $[35,36]$. It modulates the dynamics and stability of the microtubule system via its bundling and tubulin acetylation promoting activities [37,38]. These physiological functions are mediated by its direct associations with tubulin/microtubules as its primary target. In vitro TPPP/p25 is able to induce the polymerization of tubulin into normal and double-walled microtubules, tubulin aggregates and microtubule bundles as shown by electron microscopy [37] (Fig. 1). The co-localization of TPPP/p25 with the microtubule network was observed by immunofluorescence microscopy in various cell lines expressing the protein either endogenously (CG-4 cells) or after transient transfection (HeLa cells), and its bundling activity protects the microtubules against depolymerizing agents [23,38] (Fig. 1).

Mechanism by which TPPP/p25 can stabilize the microtubule network manifests itself by its dimerization capability; the dimerization induces partial folding and the exposed unstructured terminals associate with distinct microtubules resulting in their cross-linking as demonstrated by a special sandwich enzyme-linked immunosorbent assay, pelleting experiments and bimolecular fluorescence complementation assay [39].

TPPP/p25 can affect the acetylation level of the microtubule network by its direct association of the tubulin deacetylases such as histone deacetylase 6 (HDAC6) and the $\mathrm{NAD}^{+}$-dependent deacetylase sirtuin-2 (SIRT2) [38,40]; while the classic HDAC6 inhibitors, TSA and SAHA, result in maximum acetylation of the microtubule network at micromolar concentration, AGK2, a SIRT2 specific inhibitor, appears to be ineffective even at a 10-fold higher concentration (Fig. 2). Compound 9, a bis hydroxamate compound is less active than the classic inhibitors likely due to its poor permeability as demonstrated by cellular ELISA experiments [41]. Thus it was concluded that such in vitro experiments performed with HeLa cells using HDAC6 inhibitors with distinct potency are suitable for functional studies at controlled acetylation levels.

HDAC6 displays a tubulin deacetylase activity although it also modulates microtubule dynamics without altering acetylation as proved by using a catalytically inactive enzyme [42]. Moreover, HDAC6 also plays a role in the transport and autophagic clearance of misfolded proteins [43]. The integration of TPPP/p25 to the tubulin-associated HDAC6 significantly reduces the deacetylase activity, TPPP/p25 functions as a HDAC6 inhibitor (Fig. 2). However, this inhibitory potency is not originated from the release of the HDAC6 from the tubulin in the presence of TPPP/p25 rather its structural reorganization within the ternary complex [40]. The binding of TPPP/p25 to HDAC6 and/or SIRT2 as physiological 
partner proteins results in increased microtubule acetylation, in addition to its bundling activity, which influence the stability, dynamics, growth velocity of the microtubules as well as the microtubule-derived cell motility [38]. All these functions of TPPP/p25 contribute to the control of cell division; the uncontrolled cell proliferation is one of the major characteristic of the tumor progression.

The multifarious functions of TPPP/p25 are also regulated by post-translational modifications. The phosphorylation mediated by ERK2 and cyclin-dependent kinase 5 (on Thr14, Ser18 and Ser160) resulted in the loss of TPPP/p25-induced microtubule assembly [44]; LIM kinase 1-induced phosphorylation of the Ser residue(s) reduced the polymerization promoting potency of TPPP/p25 [45]. The Rho-associated coiled-coil kinase phosphorylates TPPP/p25 (on Ser32, Ser107 and Ser159), without affecting its tubulin polymerization promoting activity, however, the phosphorylation inhibits its interaction with HDAC6 resulting in increased HDAC6 activity coupled with decreased microtubule acetylation [46]. Therefore, the phosphorylation of distinct sites(s) by different kinases may play a crucial role in its multifunctional functions such as its mitotic regulatory activity [47].

TPPP/p25 is a microtubule associated disordered protein, which is related to its physiological functions; however, it displays pathological ones as well that are determined by its interacting partners ( $\alpha$-synuclein). TPPP/p25 is the prototype of Neomorphic Moonlighting Proteins [48,49] (Fig. 3). The functional binding studies with different truncated, deletion mutants and fragments of TPPP/p25 proved that the binding segment(s) of the full-length TPPP/p 25 could be replaced by other segments resulting in distinct specificities and/or binding affinities [50]. This intriguing phenomenon was denoted neomorphic chameleon feature, in this case the functional plasticity is due to alterations at gene level [50]. Obviously, the high conformational plasticity with the unique amino acid composition and sequence can ensure exceptional functional resilience.

\section{INNOVATIVE STRATEGY FOR PD THERAPY}

In addition to $\alpha$-synuclein [51], the well-established hallmark protein of PD and other synucleinopathies such as MSA, TPPP/p25 has been found to be enriched and co-localized with $\alpha$ synuclein in the pathological human inclusions functioning as a biomarker protein [27-29,52,53]. TPPP/p25 was demonstrated in Lewy bodies of substantia nigra pars compacta of LBD and PD by immunohistochemistry by confocal microscopy using highly specific mono- and polyclonal antibodies [27-30]. In addition, the presence of TPPP/p25 in isolated Lewy bodies and Lewy neurites was established by double immunofluorescence confocal laser-scanning microscopy [29]. MSA also belongs to synucleinopathies, which is characterized by glial cytoplasmic inclusions [53], in which TPPP/p25 was found to be co-localized with $\alpha$-synuclein $[27,52-54]$ in spite of the fact that this latter protein is expressed in neurons $[55,56]$. The potency of TPPP/p25 to induce oligomers and protofilaments of $\alpha$ synuclein has been reported $[29,57,58]$. Both TPPP/p25 and $\alpha$-synuclein can be taken up by HeLa and $\mathrm{CHO}$ cells from the medium resulting in their intracellular co-aggregation as detected by immunofluorescence microscopy [57,58]. The precise mechanism how the aberrant protein-protein interactions of these disordered hallmarks convey aggregates leading to the formation of pathological inclusions in brain has not been discovered in details; however, it has been accepted that TPPP/p25 is a promoting factor of the formation of pathological aggregates [29,57-59]. Yet, neither $\alpha$-synuclein nor $\mathrm{TPPP} / \mathrm{p} 25$ can be considered as an anti-Parkinson drug target since both proteins display physiological functions as well $[48,60]$. In addition, as demonstrated very recently drug targeting of moonlighting and/or chameleon proteins involved in neurodegeneration is highly challenging [50].

In rational drug design, molecular docking and crystallography have been usually applied to target globular proteins. In the cases of $\alpha$-synuclein pathology, in silico high-throughput structure-based

URL: https://mc.manuscriptcentral.com/eru Email: anna.woolman@informa.com 
docking screen method combined with experiments was suggested leading to fragment-like molecule design [61]. However, this strategy does not take into account the neomorphic moonlighting and neomorphic chameleon features of the disordered proteins involved in the inclusion formation in the case of synucleinopathies. Recently the challenges associated with targeting of chameleon proteins have been explored using the TPPP/p25- $\alpha$-synuclein complex under conditions which is unfavorable for the oligomerization as a case study [50]. The binding segments on TPPP/p25 involved in the physiological and pathological interactions have been identified at molecular and cellular levels [57,58]; accordingly the unstructured $\mathrm{C}$-terminus interacts with tubulin, while the flexible middle CORE segment associates with the C-terminus of $\alpha$-synuclein [57,58]. The dissimilarity of the binding motives involved in the physiological and pathological interactions has significant innovative impact in anti-Parkinson drug research. Therefore, the interface of the TPPP/p25- $\alpha$-synuclein complex has been recently identified and validated as a specific drug target and this innovative strategy has been suggested for anti-Parkinson drug development [50,57,58] (Fig. 4).

It is worth noting that in normal brain tissues $\alpha$-synuclein and TPPP/p25 are expressed predominantly in distinct cell types, neurons or oligodendrocytes (OLGs), respectively $[55,56,62,63]$. Originally, TPPP/p25 was identified from rat brain; it is localized predominantly in OLGs, in neuropil and fiber-like structures of the CA3 hippocampal region [62]. Subcellular proteomics revealed that besides $\alpha$-synuclein TPPP/p25 is also a component of neuromelanin granules of substantia nigra neurons [64], and it has also been found in various synaptic preparations as a component of the postsynaptic density, which suggests it may occur in neuronal cells at a very modest level [65]. Cell-tocell transmission of $\alpha$-synuclein by different mechanisms as well as the uptake of both hallmark proteins by eukaryotic cells from the media has been reported [57,66]. $\alpha$-synuclein (as well as its oligomeric or the Ser129 phosphorylated forms) has been detected in the cerebrospinal fluid of PD patients $[67,68]$. The presence of TPPP/p25 in the cerebrospinal fluid has been detected in some patients suffering from multiple sclerosis [69]. The fact that both proteins have been detected in cerebrospinal fluid [67-69] rationale their co-existence and co-aggregation in both neurons and OLGs of diseased cells. Consequently, the TPPP/p25- $\alpha$-synuclein pathological complex could be potential drug target; the interface of the complex should be targeted by competitors/foldamers to impede or to destruct the intracellular TPPP/p25- $\alpha$-synuclein complex with no or minimal side effect.

\section{UNCONTROLLED MICROTUBULE DYNAMICS AND CANCER}

$\mathrm{TPPP} / \mathrm{p} 25$ is expressed predominantly in differentiated (but not in the dividing progenitor) OLGs of normal human brain [62,70]; the differentiation is coupled with the expression of TPPP/p25 [63]. OLGs are the main constituents of the myelin sheath wrapping the axons, therefore these cells are indispensable for the proper transmission of signal along axons. Indeed, in the case of multiple sclerosis, which is a demyelinating disease, loss of TPPP/p25-positive OLGs in the brain and increased TPPP/p25 levels in the cerebrospinal fluid of patients were found, respectively [69,71]. Moreover, TPPP/p25 appears as a potential factor of mitotic process in the course of cell cycle and differentiation. In the case of glioma, a brain tumor, the expression of TPPP/p25 is negligible [72]. In agreement with this observation, the microinjection of TPPP/p25 into cleavage Drosophila embryo was found to inhibit mitotic spindle assembly and nuclear envelope breakdown without affecting other cellular events [73]. More recent data have shown that TPPP/p25 is involved in the regulation of astral microtubules and spindle orientation during mitosis [74], it reduces cell proliferation via inhibition of the $\mathrm{G}_{1} / \mathrm{S}$-phase transition and the progression of cells into $\mathrm{G}_{1}$ from $\mathrm{G}_{2} / \mathrm{M}$-phase [47]. Overexpression of TPPP/p25 was found to significantly reduce, while its RNAi-mediated knockdown significantly increased the rate of 
cell proliferation in osteosarcoma (U2OS) cell line [47]. These physiological and pathological data concern with the lack/low level of TPPP/p25 expression in cancer cells such as HeLa or neuroblastoma and suggest its anti-proliferative potency (Fig. 5). Rather surprisingly, TPPP/p20 (a homologue of TPPP/p25 lacking its $\mathrm{N}$-terminus) also displays tubulin polymerization promoting and microtubule bundling activities [75], but it is enriched in certain lung carcinomas positively associated with tumor size, metastasis, and poor survival [76,77]; its depletion suppresses the uncontrolled cell proliferation coupled with mitotic abnormalities [78].

There is a growing family of microtubule-associated proteins such as oncogenes, tumor suppressors, and apoptosis regulators that alter the microtubule dynamics which is considered as one of the critical events in tumorigenesis and tumor progression [79]. Many of them are seemingly unrelated proteins that share a common microtubule-related function, thus these microtubule associated proteins with their microtubule-stabilizing/destabilizing potency may play crucial role in the rational microtubule-targeting cancer therapy [79]. Natural and synthetic compounds that disrupt microtubule dynamics are among the most successful and widely used cancer chemotherapeutic agents [79]. There is accumulating evidence that a family of cellular proteins that are associated with and alter the dynamics of microtubules can determine the sensitivity of cancer cells to microtubule-targeting agents and play a role in tumor cell resistance to these agents; such as the pro-apoptotic BIM protein [80].

The relationship between dementia and cancer is complex and far from the agreement. There are observations based upon mostly population and case-control studies that suggest reduced risk of certain cancers and increased risk of malignant melanoma in PD [81-83]. Common factors such as aging, chronic inflammation and immunosenescence have been implicated. However, the pathophysiological pictures are further complicated by the facts that certain cancer therapy agents have neurotoxic effects, while others reduce neurodegeneration $[22,84]$. Shared genetic pathways seem to be a major focus, particularly those favoring apoptosis and cell proliferation [83]. In fact, evidence has steadily emerged on intriguing relationships between cancer and neurodegenerative diseases, both disorders of aging; however, the signals are mixed that make difficult the straightforward mechanism $[81,85]$. The mechanisms underlying these associations are far from clear, in part because of the heterogeneity of both cancer and neurodegeneration.

Molecular modulation of microtubule dynamics and stability seems to be the most effective way to affect cancerous processes as demonstrated by treatment of cancer with paclitaxel and Vinca alkaloids acting as stabilizer and destabilizer of the microtubule system, respectively. Nowadays microtubules are also promising targets in PD [84]. Epothilone D (which stabilizes microtubules and can cross the blood-brain barrier) rescued microtubule defects and diminished MPTP-induced degeneration; davunetide (a microtubule-interacting peptide) improved motor functions and reduced the aggregation of $\alpha$-synuclein in mouse model of PD [21,22]. Tyrosine kinase Abelson (c-Abl) has been implicated in human leukemia, but it also phosphorylates hallmark proteins of $\operatorname{PD~(~} \alpha$-synuclein, parkin) in dopaminergic neurons [86]. c-Abl kinase inhibitors are applied to treat cancer, and now these molecules (such as nilotinib) are also in the focus of PD therapy. Nilotinib can cross the blood-brain barrier and has been found neuroprotective in animal models of PD; it improved motor behavior of mice and also increased the concentrations of dopamine and its metabolites [87,88]. Further investigations are necessary to study its effects on dopaminergic neurons in models resembling more closely human patients.

The anti-proliferative potency of TPPP/p25, hallmark protein of synucleinopathies, may raise its influence also in cancer development. The recognition that whereas too much TPPP/p25 could kill neurons in $\mathrm{PD}$, but its loss keeps cells alive in cancer could contribute to our understanding of the 
interrelationship of "TPPP/p25 diseases". Therefore, the deeper understanding of its physiological and pathological interactions and regulation of its expression levels is of great importance.

\section{EXPERT COMMENTARY}

Although the motor symptoms of PD can be alleviated by using various therapies; still, no neuroprotective or neurorestorative therapy exists for the treatment of this chronic disorder [3,4,21,33]. The discovery and development of therapeutic strategies for the treatments of PD and other CNS diseases have been limited by a lack of understanding of the mechanisms driving protein aggregation and inclusion body formation which result in progressive dopaminergic cell loss. Hallmark proteins of the conformational diseases such as PD are usually disordered proteins without well-defined 3D structures. These proteins, such as TPPP/p25, often display moonlighting and chameleon features $[48,50,89,90]$, thus the classic bioinformatics (e.g. docking) and experimental methods (e.g. crystallography) are inefficient to develop specific drugs with negligible side-effects. Disordered proteins as drug targets have been rarely studied, but novel experimental and computational methods are emerging to investigate their druggability [91,92]. Targeting of neomorphic moonlighting proteins is a challenging task since the pathological interactions should be prevented/impeded without influencing the physiological ones. This issue has not been taken into consideration in most of the Parkinson research.

In a case study focusing on the TPPP/p25- $\alpha$-synuclein complex, the potential initiator of the inclusion formation, the dissimilarity of TPPP/p 25 binding segments involved in its physiological and pathological interactions could solve the specificity of drug targeting as a new innovative strategy for anti-Parkinson drug development [57,58]. The interfaces of the TPPP/p25 complexed with tubulin and $\alpha$-synuclein have been recently identified and validated at molecular and cellular levels [57,58]; nevertheless, multinuclear NMR studies would contribute to the identification of the segments at atomic level that renders it possible the design and development of specific drugs such as peptidomimetic foldamers with specific foldamer potency.

\section{FIVE-YEAR PERSPECTIVE}

A new, exciting and emerging area is the relationship of neurological disorders such as PD with cancer [81-83]. At first, it is hard to imagine two diseases more different than PD and cancer. Tumorigenesis and neurodegeneration have been described as two sides of the same coin [93], and cancer (abnormal cell proliferation) and neurodegeneration (abnormal cell death) can be considered as the opposite ends of a spectrum regarding genes which control the cell cycle [81]. TPPP/p25, besides its potency to induce $\alpha$-synuclein aggregation, displays anti-proliferative (anti-mitotic) activity as well $[47,63,72,73]$. Therefore, it seems that the uncontrolled (non-physiological) level of TPPP/p25 leads to distinct diseases: its enrichment leads to PD and other synucleinopathies due to overexpression [27$30,52,53]$, while its low level or lack is coupled with uncontrolled cell division characteristic for glioma, a brain tumor [72]. In the years to come, our ability to understand the interrelationship of "TPPP/p25 diseases" is expected to grow, and this new area of the medical sciences should be in the focus of the near future researches.

\section{KEY ISSUES}

URL: https://mc.manuscriptcentral.com/eru Email: anna.woolman@informa.com 
- The discovery and development of therapeutic strategies for the treatments of Parkinson's disease (PD) and other synucleinopathies are limited by a lack of understanding of the pathomechanisms driving protein aggregation and inclusion body formation leading to progressive dopaminergic cell loss.

- The hallmarks of these diseases are frequently disordered proteins displaying unique (neomorphic) moonlighting and/or chameleon features, which are challenging drug targets.

- The disordered Tubulin Polymerization Promoting Protein (TPPP/p25), a neomorphic moonlighting protein, displays distinct functions by day and at night; it modulates the dynamics and stability of the microtubule system at physiological condition via its bundling and tubulin acetylation promoting activities; however, at pathological conditions it is either co-enriched and co-localized with $\alpha$-synuclein (in neurodegeneration) or omitted (in cancer).

- As a new innovative strategy in the Parkinson research, the interface segments of TPPP/p25- $\alpha$ synuclein complex has been validated as potential drug target.

- The recognition that whereas too much TPPP/p25 could kill neurons leading to neurodegeneration, while its loss keeps cells alive in cancer suggesting its anti-proliferative potency; thus these diseases could be classified as "TPPP/p25 diseases".

\section{LIST OF ABBREVIATIONS}

dementia with Lewy bodies, LBD; histone deacetylase 6, HDAC6; multiple system atrophy, MSA; 1-methyl-4-phenyl-1,2,3,6-tetrahydropyridine, MPTP; oligodendrocyte, OLG; Parkinson's disease, PD; $\mathrm{NAD}^{+}$-dependent deacetylase sirtuin-2, SIRT2; Tubulin Polymerization Promoting Protein, $\mathrm{TPPP} / \mathrm{p} 25$.

\section{DECLARATION OF INTERESTS}

The authors report no conflicts of interest.

\section{REFERENCES}

* Of interest

** Of considerable interest

1. Lashuel HA, Overk CR, Oueslati A, et al. The many faces of $\alpha$-synuclein: from structure and toxicity to therapeutic target. Nat Rev Neurosci. 2013;14(1):38-48.

2. Zhang H, Tong R, Bai L, et al. Emerging targets and new small molecule therapies in Parkinson's disease treatment. Bioorg Med Chem. 2016;24(7):1419-1430.

3. Lindholm D, Mäkelä J, Di Liberto V, et al. Current disease modifying approaches to treat Parkinson's disease. Cell Mol Life Sci. 2016;73(7):1365-1379.

4. Oertel W, Schulz JB. Current and experimental treatments of Parkinson disease: A guide for neuroscientists. J Neurochem. 2016;139 Suppl 1:325-337. 
5. Bellucci A, Mercuri NB, Venneri A, et al. Review: Parkinson's disease: fro synaptic loss to connectome dysfunction. Neuropathol Appl Neurobiol. 2016;42(1):77-94.

6. Bennett MC, Bishop JF, Leng Y, et al. Degradation of alpha-synuclein by proteasome. J Biol Chem. 1999;274(48):33855-33858.

7. Webb JL, Ravikumar B, Atkins J, et al. Alpha-Synuclein is degraded by both autophagy and the proteasome. J Biol Chem. 2003;278(27):25009-25013.

8. Cuervo AM, Stefanis L, Fredenburg R, et al. Impaired degradation of mutant alpha-synuclein by chaperone-mediated autophagy. Science. 2004;305(5688):1292-1295.

9. Dehay B, Bové J, Rodríguez-Muela N, et al. Pathogenic lysosomal depletion in Parkinson's disease. J Neurosci. 2010;30(37):12535-12544.

10. Chu Y, Dodiya H, Aebischer P, et al. Alterations in lysosomal and proteasomal markers in Parkinson's disease: relationship to alpha-synuclein inclusions. Neurobiol Dis. 2009;35(3):385398.

11. Spillantini MG, Schmidt ML, Lee VM, et al. Alpha-synuclein in Lewy bodies. Nature. 1997;388(6645):839-840.

12. Del Tredici K, Braak H. Review: Sporadic Parkinson's disease: development and distribution of $\alpha$-synuclein pathology. Neuropathol Appl Neurobiol. 2016;42(1):33-50.

13. Parkkinen L, Pirttilä T, Alafuzoff I. Applicability of current staging/categorization of alphasynuclein pathology and their clinical relevance. Acta Neuropathol. 2008;115(4):399-407.

14. Rey NL, George S, Brundin P. Review: Spreading the word: precise animal models and validated methods are vital when evaluating prion-like behaviour of alpha-synuclein. Neuropathol Appl Neurobiol. 2016;42(1):51-76.

15. Winner B, Jappelli R, Maji SK, et al. In vivo demonstration that alpha-synuclein oligomers are toxic. Proc Natl Acad Sci U S A. 2011;108(10):4194-4199.

16. Polymeropoulos MH, Lavedan C, Leroy E, et al. Mutation in the alpha-synuclein gene identified in families with Parkinson's disease. Science. 1997;276(5321):2045-2047.

17. Okochi M, Walter J, Koyama A, et al. Constitutive phosphorylation of the Parkinson's disease associated alpha-synuclein. J Biol Chem. 2000;275(1):390-397.

18. Fujiwara H, Hasegawa $M$, Dohmae $N$, et al. alpha-Synuclein is phosphorylated in synucleinopathy lesions. Nat Cell Biol. 2002;4(2):160-164.

19. Conde C, Cáceres A. Microtubule assembly, organization and dynamics in axons and dendrites. Nat Rev Neurosci. 2009;10(5):319-332.

20. de Forges H, Bouissou A, Perez F. Interplay between microtubule dynamics and intracellular organization. Int J Biochem Cell Biol. 2012;44(2):266-274.

21. Cartelli D, Cappelletti G. Microtubule Destabilization Paves the Way to Parkinson's Disease. Mol Neurobiol. 2016 [2016 Oct 18]; [13]. DOI: 10.1007/s12035-016-0188-5.

22. Pellegrini L, Wetzel A, Grannó S, et al. Back to the tubule: microtubule dynamics in Parkinson's disease. Cell Mol Life Sci. 2016 [2016 Sep 6]; [26]. DOI: 10.1007/s00018-016-2351-6.

23. Lehotzky A, Tirián L, Tökési N, et al. Dynamic targeting of microtubules by TPPP/p25 affects cell survival. J Cell Sci. 2004;117(Pt 25):6249-6259.

24. Kopito RR. Aggresomes, inclusion bodies and protein aggregation. Trends Cell Biol. 2000;10(12):524-530.

25. Cartelli D, Aliverti A, Barbiroli A, et al. $\alpha$-Synuclein is a Novel Microtubule Dynamase. Sci Rep. 2016;6:33289.

26. Wakabayashi K, Tanji K, Odagiri S, et al. The Lewy body in Parkinson's disease and related neurodegenerative disorders. Mol Neurobiol. 2013;47(2):495-508. 
27. Kovács GG, László L, Kovács J, et al. Natively unfolded tubulin polymerization promoting protein TPPP/p25 is a common marker of alpha-synucleinopathies. Neurobiol Dis. 2004;17(2):155-162.

** This was the first study which proved the co-localization of TPPP/p25 and a-synuclein in inclusion bodies characteristic for PD and MSA suggested TPPP/p25 as a hallmark protein of synucleinopathies.

28. Kovács GG, Gelpi E, Lehotzky A, et al. The brain-specific protein TPPP/p25 in pathological protein deposits of neurodegenerative diseases. Acta Neuropathol. 2007;113(2):153-161.

29. Lindersson E, Lundvig D, Petersen C, et al. p25alpha Stimulates alpha-synuclein aggregation and is co-localized with aggregated alpha-synuclein in alpha-synucleinopathies. J Biol Chem. 2005;280(7):5703-5715.

30. Jellinger KA. P25alpha immunoreactivity in multiple system atrophy and Parkinson disease. Acta Neuropathol. 2006;112(1):112.

31. Moussaud S, Jones DR, Moussaud-Lamodière EL, et al. Alpha-synuclein and tau: teammates in neurodegeneration? Mol Neurodegener. 2014;9:43.

32. Tsigelny IF, Crews L, Desplats P, et al. Mechanisms of hybrid oligomer formation in the pathogenesis of combined Alzheimer's and Parkinson's diseases. PLoS One. 2008;3(9):e3135.

33. Dehay B, Bourdenx M, Gorry P, et al. Targeting $\alpha$-synuclein for treatment of Parkinson's disease: mechanistic and therapeutic considerations. Lancet Neurol. 2015;14(8):855-866.

34. Swart C, Haylett W, Kinnear C, et al. Neurodegenerative disorders: dysregulation of a carefully maintained balance? Exp Gerontol. 2014;58:279-291.

35. Orosz F, Kovács GG, Lehotzky A, et al. TPPP/p25: from unfolded protein to misfolding disease: prediction and experiments. Biol Cell. 2004;96(9):701-711.

36. Zotter A, Bodor A, Oláh J, et al. Disordered TPPP/p25 binds GTP and displays Mg2+-dependent GTPase activity. FEBS Lett. 2011;585(5):803-808.

37. Hlavanda E, Kovács J, Oláh J et al. Brain-specific p 25 protein binds to tubulin and microtubules and induces aberrant microtubule assemblies at substoichiometric concentrations. Biochemistry. 2002;41(27):8657-8664.

38. Tőkési N, Lehotzky A, Horváth I, et al. TPPP/p25 promotes tubulin acetylation by inhibiting histone deacetylase 6. J Biol Chem. 2010;285(23):17896-17906.

39. Oláh J, Szénási T, Szunyogh S, et al. Further evidence for microtubule-independent dimerization of TPPP/p25. Sci Rep. 2017;7:40594.

40. Mangas-Sanjuan V, Oláh J, Gonzalez-Alvarez I, et al. Tubulin acetylation promoting potency and absorption efficacy of deacetylase inhibitors. Br J Pharmacol. 2015;172(3):829-840.

41. Zwick V, Nurisso A, Simões-Pires C, et al. Cross metathesis with hydroxamate and benzamide BOC-protected alkenes to access HDAC inhibitors and their biological evaluation highlighted intrinsic activity of BOC-protected dihydroxamates. Bioorg Med Chem Lett. 2016;26(1):154-159.

42. Zilberman Y, Ballestrem C, Carramusa Let al. Regulation of microtubule dynamics by inhibition of the tubulin deacetylase HDAC6. J Cell Sci. 2009;122(Pt 19):3531-3541.

43. Pandey UB, Nie Z, Batlevi Y, et al. HDAC6 rescues neurodegeneration and provides an essential link between autophagy and the UPS. Nature. 2007;447(7146):859-863.

44. Hlavanda E, Klement E, Kókai E, et al. Phosphorylation blocks the activity of tubulin polymerization-promoting protein (TPPP): identification of sites targeted by different kinases. $\mathrm{J}$ Biol Chem. 2007;282(40):29531-29539.

45. Acevedo K, Li R, Soo P, et al. The phosphorylation of p25/TPPP by LIM kinase 1 inhibits its ability to assemble microtubules. Exp Cell Res. 2007313(20):4091-4106.

URL: https://mc.manuscriptcentral.com/eru Email: anna.woolman@informa.com 
46. Schofield AV, Steel R, Bernard O. Rho-associated coiled-coil kinase (ROCK) protein controls microtubule dynamics in a novel signaling pathway that regulates cell migration. J Biol Chem. 2012;287(52):43620-43629.

47. Schofield AV, Gamell C, Suryadinata R, et al. Tubulin polymerization promoting protein 1 (Tppp1) phosphorylation by Rho-associated coiled-coil kinase (rock) and cyclin-dependent kinase 1 (Cdk1) inhibits microtubule dynamics to increase cell proliferation. J Biol Chem. 2013;288(11):7907-7917.

48. Ovádi J. Moonlighting proteins in neurological disorders. IUBMB Life. 2011;63(7):453-456.

49. Jeffery CJ. Proteins with neomorphic moonlighting functions in disease. IUBMB Life. 2011;63(7):489-494.

50. Szénási T, Oláh J, Szabó A, et al. Challenging drug target for Parkinson's disease: Pathological complex of the chameleon TPPP/p25 and alpha-synuclein proteins. Biochim Biophys Acta. 2017;1863(1):310-323.

* This study provided evidence for the chameleon feature of TPPP/p25.

51. Goedert M, Spillantini MG, Del Tredici K, et al. 100 years of Lewy pathology. Nat Rev Neurol. 2013;9(1):13-24.

52. Ota K, Obayashi M, Ozaki K, et al. Relocation of $\mathrm{p} 25 \alpha /$ tubulin polymerization promoting protein from the nucleus to the perinuclear cytoplasm in the oligodendroglia of sporadic and COQ2 mutant multiple system atrophy. Acta Neuropathol Commun. 2014;2:136.

53. Jellinger KA, Wenning GK. Multiple system atrophy: pathogenic mechanisms and biomarkers. J Neural Transm (Vienna). 2016;123(6):555-572.

54. Hasegawa T, Baba T, Kobayashi M, et al. Role of TPPP/p25 on $\alpha$-synuclein-mediated oligodendroglial degeneration and the protective effect of SIRT2 inhibition in a cellular model of multiple system atrophy. Neurochem Int. 2010;57(8):857-866.

55. Maroteaux L1, Campanelli JT, Scheller RH. Synuclein: a neuron-specific protein localized to the nucleus and presynaptic nerve terminal. J Neurosci. 1988;8(8):2804-2815.

56. Bates CA, Zheng W. Brain disposition of $\alpha$-Synuclein: roles of brain barrier systems and implications for Parkinson's disease. Fluids Barriers CNS. 2014;11:17.

57. Tökési N, Oláh J, Hlavanda E, et al. Identification of motives mediating alternative functions of the neomorphic moonlighting TPPP/p25. Biochim Biophys Acta. 2014;1842(4):547-557.

** This study suggested a new innovative strategy for PD, and validated the interface of the moonlighting TPPP involved in its pathological interactions as a drug target.

58. Szunyogh S, Oláh J, Szénási T, et al. Targeting the interface of the pathological complex of $\alpha$ synuclein and TPPP/p25. Biochim Biophys Acta. 2015;1852(12):2653-2661.

59. Song YJ, Lundvig DM, Huang Y, et al. p25alpha relocalizes in oligodendroglia from myelin to cytoplasmic inclusions in multiple system atrophy. Am J Pathol. 2007;171(4):1291-1303.

60. Dettmer U, Selkoe D, Bartels T. New insights into cellular $\alpha$-synuclein homeostasis in health and disease. Curr Opin Neurobiol. 2016;36:15-22.

61. Tóth G, Gardai SJ, Zago W, et al. Targeting the intrinsically disordered structural ensemble of $\alpha$ synuclein by small molecules as a potential therapeutic strategy for Parkinson's disease. PLoS One. 2014;9(2):e87133.

62. Takahashi M, Tomizawa K, Fujita SC, et al. A brain-specific protein p25 is localized and associated with oligodendrocytes, neuropil, and fiber-like structures of the CA3 hippocampal region in the rat brain. J Neurochem. 1993;60(1):228-235.

63. Lehotzky A, Lau P, Tökési N, et al. Tubulin polymerization-promoting protein (TPPP/p25) is critical for oligodendrocyte differentiation. Glia. 2010;58(2):157-168. 
64. Tribl F, Gerlach M, Marcus K, et al. "Subcellular proteomics" of neuromelanin granules isolated from the human brain. Mol Cell Proteomics. 2005;4(7):945-957.

65. Orosz F, Lehotzky A, Oláh J, Ovádi J. 2009. TPPP/p25: A new unstructured protein hallmarking synucleinopathies. In: Ovádi J, Orosz F, editors. Protein Folding and Misfolding: Neurodegenerative Diseases. New York (NY): Springer-Verlag. pp 225-250.

66. Danzer KM, Kranich LR, Ruf WP, et al. Exosomal cell-to-cell transmission of alpha synuclein oligomers. Mol Neurodegener. 2012;7:42.

67. Marques O, Outeiro TF. Alpha-synuclein: from secretion to dysfunction and death. Cell Death Dis. 2012;3:e350.

68. Andersen AD, Binzer M, Stenager E, Gramsbergen JB. Cerebrospinal fluid biomarkers for Parkinson's disease - a systematic review. Acta Neurol Scand. 2017;135(1):34-56.

69. Vincze O, Oláh J, Zádori D, et al. A new myelin protein, TPPP/p25, reduced in demyelinated lesions is enriched in cerebrospinal fluid of multiple sclerosis. Biochem Biophys Res Commun. 2011;409(1):137-141.

70. Skjoerringe T, Lundvig DM, Jensen $\mathrm{PH}$, et al. P25alpha/Tubulin polymerization promoting protein expression by myelinating oligodendrocytes of the developing rat brain. J Neurochem. 2006;99(1):333-342.

71. Höftberger R, Fink S, Aboul-Enein F, et al. Tubulin polymerization promoting protein (TPPP/p25) as a marker for oligodendroglial changes in multiple sclerosis. Glia. 2010;58(15):1847-1857.

72. Preusser M, Lehotzky A, Budka H, et al. TPPP/p25 in brain tumours: expression in nonneoplastic oligodendrocytes but not in oligodendroglioma cells. Acta Neuropathol. 2007;113(2):213-215.

73. Tirián L, Hlavanda E, Oláh $\mathrm{J}$, et al. TPPP/p25 promotes tubulin assemblies and blocks mitotic spindle formation. Proc Natl Acad Sci U S A. 2003;100(24):13976-13981.

74. Heng YW, Lim HH, Mina T, et al. TPPP acts downstream of RhoA-ROCK-LIMK2 to regulate astral microtubule organization and spindle orientation. J Cell Sci. 2012;125(Pt 6):1579-1590.

75. Vincze O, Tökési N, Oláh J, et al. Tubulin polymerization promoting proteins (TPPPs): members of a new family with distinct structures and functions. Biochemistry. 2006;45(46):13818-13826.

76. Zhou W, Li J, Wang X, et al. Stable knockdown of TPPP3 by RNA interference in Lewis lung carcinoma cell inhibits tumor growth and metastasis. Mol Cell Biochem. 2010;343(1-2):231-238.

77. Li Y, Xu Y, Ye K, et al. Knockdown of Tubulin Polymerization Promoting Protein Family Member 3 Suppresses Proliferation and Induces Apoptosis in Non-Small-Cell Lung Cancer. J Cancer. 2016;7(10):1189-1196.

78. Zhou W, Wang X, Li L, et al. Depletion of tubulin polymerization promoting protein family member 3 suppresses HeLa cell proliferation. Mol Cell Biochem. 2010;333(1-2):91-98.

79. Bhat KM, Setaluri V. Microtubule-associated proteins as targets in cancer chemotherapy. Clin Cancer Res. 2007;13(10):2849-2854.

80. Luo S, Rubinsztein DC. BCL2L11/BIM: a novel molecular link between autophagy and apoptosis. Autophagy. 2013;9(1):104-105.

81. Ganguli M. Cancer and Dementia: It's Complicated. Alzheimer Dis Assoc Disord. 2015;29(2):177-182.

82. Feng DD, Cai W, Chen X. The associations between Parkinson's disease and cancer: the plot thickens. Transl Neurodegener. 2015;4:20.

83. Plun-Favreau H, Lewis PA, Hardy J, et al. Cancer and neurodegeneration: between the devil and the deep blue sea. PLoS Genet. 2010;6(12):e1001257.

URL: https://mc.manuscriptcentral.com/eru Email: anna.woolman@informa.com 
84. Eira J, Silva CS, Sousa MM, et al. The cytoskeleton as a novel therapeutic target for old neurodegenerative disorders. Prog Neurobiol. 2016;141:61-82.

85. Catalá-López F, Suárez-Pinilla M, Suárez-Pinilla $\mathrm{P}$, et al. Inverse and direct cancer comorbidity in people with central nervous system disorders: a meta-analysis of cancer incidece in 577,013 participants of 50 observational studies. Psychother Psychosom. 2014;83(2):89-105.

86. Lindholm D, Pham DD, Cascone A, et al. c-Abl Inhibitors Enable Insights into the Pathophysiology and Neuroprotection in Parkinson's Disease. Front Aging Neurosci. 2016;8:254.

87. Hebron ML, Lonskaya I, Moussa CE. Nilotinib reverses loss of dopamine neurons and improves motor behavior via autophagic degradation of $\alpha$-synuclein in Parkinson's disease models. Hum Mol Genet. 2013;22(16):3315-3328.

88. Karuppagounder SS, Brahmachari S, Lee Y, et al. The c-Abl inhibitor, nilotinib, protects dopaminergic neurons in a preclinical animal model of Parkinson's disease. Sci Rep. 2014;4:4874.

89. Uversky VN. A protein-chameleon: conformational plasticity of alpha-synuclein, a disordered protein involved in neurodegenerative disorders. J Biomol Struct Dyn. 2003;21(2):211-234.

90. Silva BA, Breydo L, Uversky VN. Targeting the chameleon: a focused look at $\alpha$-synuclein and its roles in neurodegeneration. Mol Neurobiol. 2013;47(2):446-459.

91. Joshi P, Vendruscolo M. Druggability of Intrinsically Disordered Proteins. Adv Exp Med Biol. 2015;870:383-400.

92. Zhang Y, Cao H, Liu Z. Binding cavities and druggability of intrinsically disordered proteins. Protein Sci. 2015;24(5):688-705.

93. Staropoli JF. Tumorigenesis and neurodegeneration: two sides of the same coin? Bioessays. 2008;30(8):719-727.

94. Jeffery CJ. Moonlighting proteins. Trends Biochem Sci. 1999;24(1):8-11.

\section{FIGURE LEGENDS}

Figure 1. Effect of TPPP/p25 on the organization and functions of the microtubule system. A and B: electron microscopic images of TPPP/p25-promoted assembly and bundling of microtubules [73]. Bar is $100 \mathrm{~nm}$ in A and $50 \mathrm{~nm}$ in B. C: TPPP/p25 (green) aligned along the microtubule network (red) at low (1) and high (2) expression levels [23], bar is $10 \mu \mathrm{m}$. D and E: Inhibition of mitotic spindle formation in tubulin-GFP-expressing cleavage Drosophila embryo by TPPP/p25 microinjection at the posterior pole (right) [73]. A-B and D-E are modified from [73], Copyright (2003) National Academy of Sciences.

Figure 2. Relationship of HDAC6 inhibition and the acetylation level of the microtubule system. A: Scheme of the consequence of the interaction of HDAC6 with TPPP $/ \mathrm{p} 25$. B: Effect of deacetylase inhibitors on the intracellular microtubule acetylation (cellular ELISA) [40,41]. TSA: Trichostatin A, SAHA: suberoyl anilide hydroxy amide, $9: \mathrm{N}^{1}, \mathrm{~N}^{8}$-dihydroxyoctanediamide [41], AGK2: 2-Cyano-3-[5(2,5-dichlorophenyl)-2-furanyl]-N-5-quinolinyl-2-propenamide. C: Microtubule network is not acetylated in HeLa cells that do not express TPPP/p25 [38]. D: The microtubule network is acetylated in CG-4 (oligodendrocyte) cells that express TPPP/p25 endogenously [38].

Figure 3. TPPP/p25 is a neomorphic moonlighting and a neomorphic chameleon protein.

Categories of the multi-structural and multi-functional proteins: moonlighting protein (MP): displays multiple independent functions without alteration at gene level [94]; neomorphic MP: distinct 
functions at physiological and pathological conditions [48,49]; chameleon protein (CP): high conformational plasticity at protein level $[89,90]$; neomorphic $\boldsymbol{C P}$ : functional plasticity by alteration at gene level [50].

Figure 4. Potential targets for development of specific drugs for Parkinson's disease and cancer.

Figure 5. Interrelationship between neurological disorders and cancer type diseases. MT:

microtubule, SM: multiple sclerosis. 
1

2

3

4

5

6

7

9

10

11

12

13

14

15

16

17

18

19

20

21

22

23

24

25

26

27

28

29

30

31

32

33

34

35

36

37

38

39

40

41

42

43

44

45

46

47

48

49

50

51

52

53

54

55

56

57

58

59

60

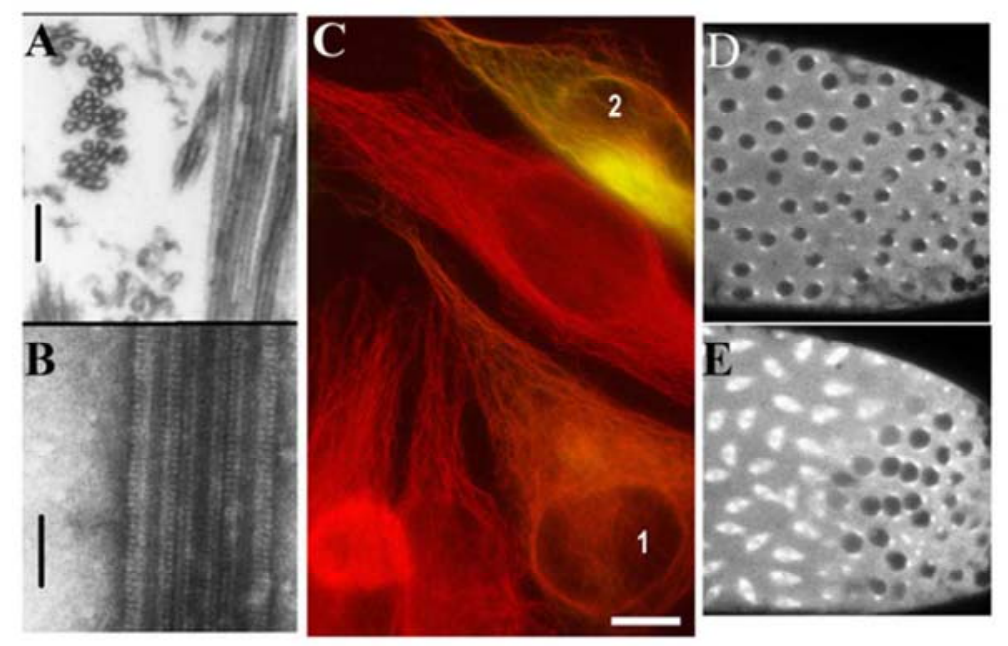

Figure 1

$59 \times 32 \mathrm{~mm}(300 \times 300 \mathrm{DPI})$ 
A
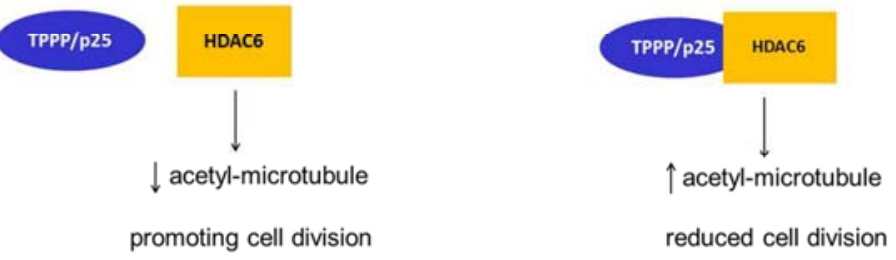

B

Effect of tubulin deacetylase inhibitors
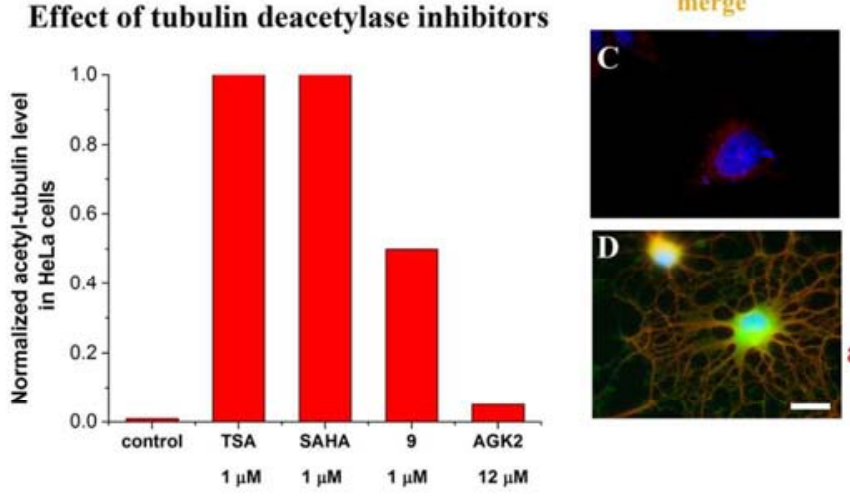

HeLa cells no TPPP/p25 expression no acetyl-microtubules

differentiated CG-4 cells endogenous TPPP/p25 acetylated microtubule network

Figure 2

$129 \times 99 \mathrm{~mm}(300 \times 300 \mathrm{DPI})$

URL: https://mc.manuscriptcentral.com/eru Email: anna.woolman@informa.com 
Figure 3

$170 \times 84 \mathrm{~mm}(300 \times 300 \mathrm{DPI})$

distinct functions at physiological and pathological conditions multiple, independent functions no changes at gene level

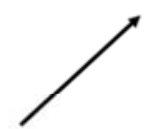

TPPP/p25

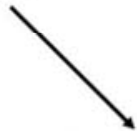

chameleon protein high conformational plasticity no changes at protein level changes at gene level deletion mutants
NEOMORPHIC

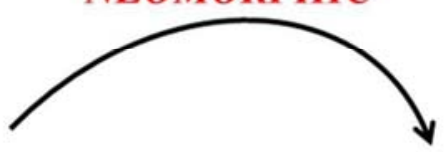

delion mutants 


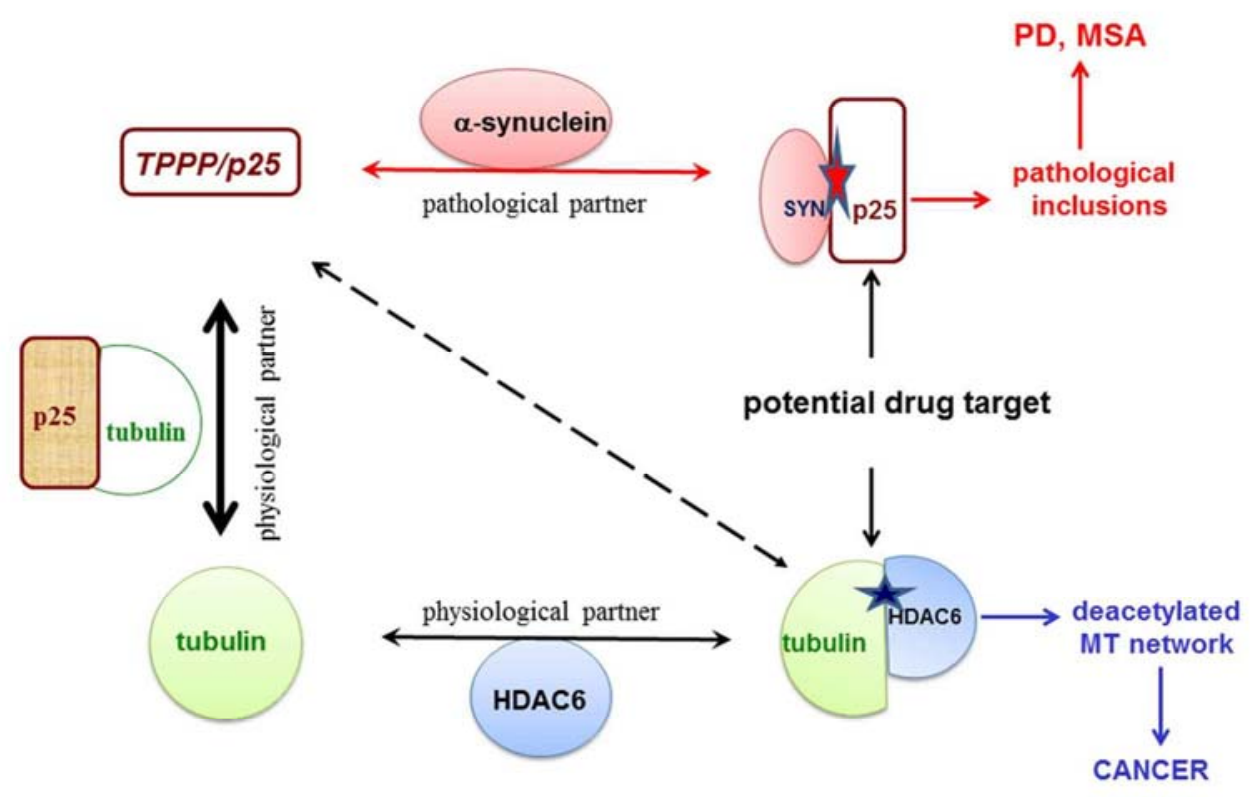

Figure 4

$170 \times 114 \mathrm{~mm}(300 \times 300$ DPI $)$ 


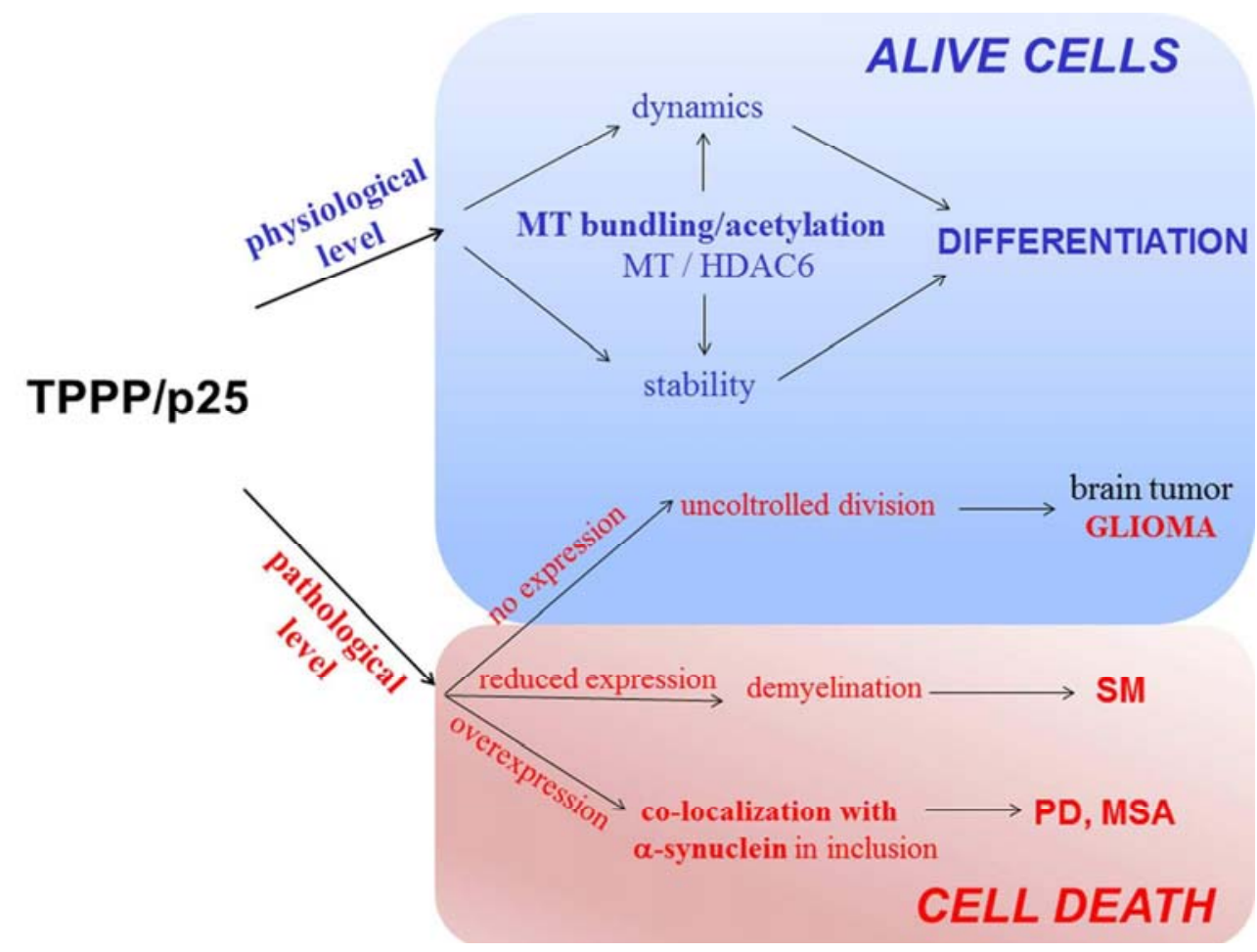

Figure 5

$170 \times 127 \mathrm{~mm}(300 \times 300 \mathrm{DPI})$ 